\title{
Learning on Tools Used in IoT Development Life Cycle
}

\author{
Shilpa V, Vidya A, S N Chandrashekara
}

\begin{abstract}
Internet of thing is an entity of the physical or virtual object, which is able to identified as well as integrated into communication system. Managing the Internet of Things is called as Web of Things. The IoT gadgets are rooted with sensors, gateways, Internet connectivity and cloud. Also using these gadgets we can converse with other devices through Internet for secured communication. Growth of IoT can be seen extremely fast in our present life. It is acknowledged that by 2020 thousands of billions of objects will be deployed globally. We trust to facilitate IoT as software-driven, therefore utility requirements resolve the modernization as well as improvement towards IoT. Primary domains identified are energy transportation, distribution, smart town, smart communication, smart domestic, atmosphere, supply chain, as well as fitness care. This study presents open source tools used in IoT development life cycle. The expression open source was mainly associated to infrastructure software, where we can improve the code re-usability rather than the implementation using web of objects.
\end{abstract}

Keywords : AMQP, CoAP, IoT, JSON, Node-RED, VSCP

\section{INTRODUCTION}

IoT is growing rapidly in everyday at a rate that is extremely quick. This brings the revise of IoT as well as its associated prospect incredibly essential. This study presents a developer to utilize the open tools for all IoT services. The word open source is associated with multiple packages in the software, that presents the original source object code. In general open source tools can be accessed from the developers till the end users to examine, utilization as well as change or growth. The development towards the Web of Things is mainly supported based on the improvement that are continuous in the area of microelectronics, IT Infrastructure. The modren days IoT application layer as shown in Fig. 1 is named as connectivity terminologies. The word connectivity mainly concentrates on Web of Things with different building blocks such as Gateway WoT, Note WoT, LAN and WAN WoT and many more. It has assistance to create more requests that are riveting as well as ranging from machine-driven entities to information analyzers.

Revised Manuscript Received on December 22, 2019.

Shilpa $\mathbf{V}^{\mathbf{1}}$, Dept of CSE,Vivekananda Institute of Technology,Bangalore, Affiliated to Visvesvaraya Technological University, Belagavi, Karnataka, Email: shilpavraghuram@gmail.com

Vidya $\mathbf{A}^{\mathbf{2}}$,Dept of CSE,Vivekananda Institute of Technology,Bangalore, Affiliated to Visvesvaraya Technological University, Belagavi, Karnataka, Email: vidyaananth16@gmail.com.

S N Chandrashekara ${ }^{3}$,Dept of CSE,CByregowda Institute of Technology,Kolar, Affiliated to Visvesvaraya Technological University, Belagavi, Karnataka, Email: hodcse.cbit2009@gmail.com

Web of Things consists of interconnected gadgets similar to smart home appliances, equipment, wheels. The user experience depends on the virtual structure as well as its specifications [1] [2] [3].

\section{UTENSILS FOR DEVELOPING INTERNET OF THINGS}

Implementing an IoT typically calls for the mutual super vision of an actuator and an endpoint. The mechanism that monitors the connected device is mainly probing for a particular assessment that energizes the end point into movement. This can be a linked home surroundings gadget that permits the end user to reveal the high temperature of the residence and alter the thermostat settings slightly, or else it can be a security gadget that tracks moves inside a structure and alert designated customers for changes.

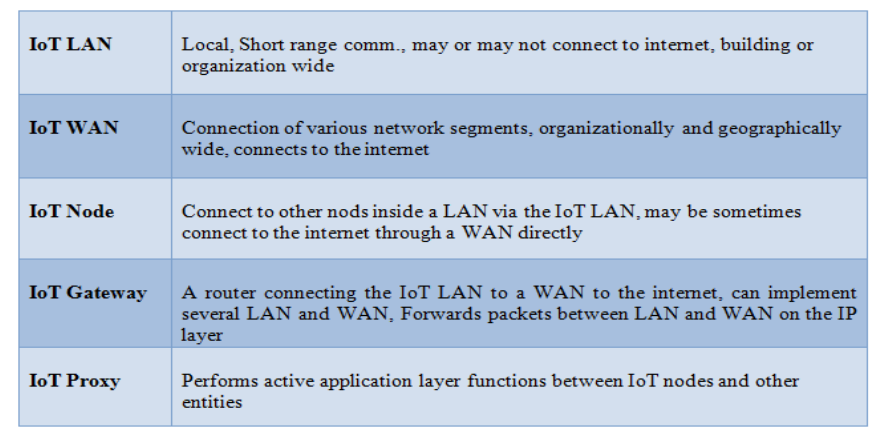

Fig. 1. Modern Days IoT Application Layer [1].

Developing applications for linked gadgets requires the following:

- Endpoint authentication,

- Session advent or creating a session,

- Destructing a session and logout from a session,

- Individual device statistics plan info,

- Individual person billing info as wished,

- Individual device details,

- User latest API activity,

- Tool claiming and activation,

- Incoming and outgoing SMS control,

- Device ordering.

IoT solutions discriminates mainstream tools provided by the company and it provides the principal edge to the IoT gadgets. There are companies that still function largely far away from the tech segment. They will also notice profit based on some market terms and conditions. 
The companies will get acknowledgement and product uniqueness when victorious smart scheme is launched. By concerning the accurate capacity it is essencial to create important solutions to facilitate the advertisements [4] [5].

\section{WHAT DRIVES OPEN RESOURCE}

The event of the open resource consists of 3 main funda- mentals such as Beneficiaries, IoT facility render, Developers [6].

- Beneficiaries: Desire to utilize large range of customer applications but not immediately those from a specific trader.

- IoT facility render: Encompass industrial various technologies, concrete the technique to resourceful compati- bilities.

- Developers: Implement freely available coding system supporting a variety of gadgets, technologies as well as different platforms rather locking with single vender.

\section{HARDWARE TOOLS FOR IOT LIFE CYCLE}

A standard existence cycle for IoT tool describes the stages of IoT gadget from beginning to end i.e., construction/reconstruction till the shutdown. Mutually both the activities have to follow the universal invention development life cycle of system requirement, system design, system development as well as testing to ensure the functional requirements. In this section, we have listed some of the hardware tools used in IoT development life cycle.

\section{A. Arduino}

Arduino is a platform-independent as well as single-board microcontroller product used equaly for both hardware as well as software as shown in Fig. 2. It is a free platform policy with a simple scheduling language and Arduino package IDE. By notifying the Ardunio board one can notice the commands to take care by providing a particular fixed instructions to a microcontroller board. For instance, Arduuno is a key deivce to examine new objects. This can be used by artists, musicians, children, programmers, hobbyists, professionals to build an interactive prototype which help to work on instrallation and testing with novel gadgets. These can also contribute ideas online with previous participants of the Arduino network. [6] [7] [8].

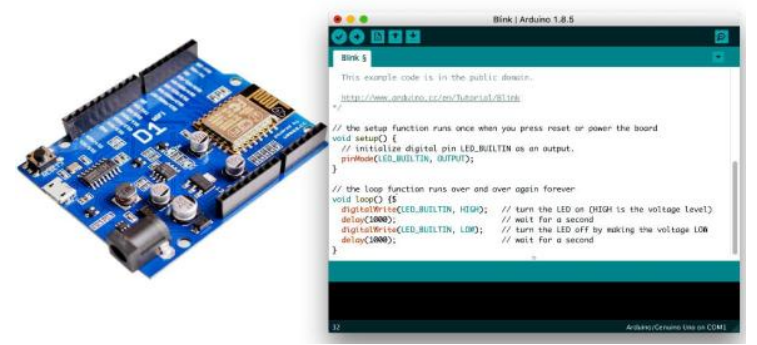

Fig. 2. Arduino Board and Arduino IDE[Integrated Development Environment] [5]
Kinoma is an hardware prototyping platform for Marvell Semiconductor. Marvell Semiconductor is a software engineering team presenting an open-source, cross-platform ECMAScript aimed at developing software program for Internet of Things, merchandise or lousy embedded devices. Kinoma mainly controls three different projects which are freely available. The Kinoma device is a DIY production kit used for electronic devices. Kinoma visual Framework/Studio is used for creating the program interface to the hardware, this can also be used for iOS and Android devises that connects smart phones and tablets. Kinoma Cerates hardware consists of an energy-efficient $\mathrm{CPU}, \mathrm{WiFi}$ and Bluetooth property, an electrical touchscreen as well as many input/output gadgets. Additionally, Kinoma provides hardware prototyping flooring aimed at the impartial architect community [6] [9] [10].

\section{The Eclipse IoT}

The Eclipse IoT functioning cluster is an assistance on manufacturing as well as intellectual partners. This technology is freely available in market and that can be used by busi- ness/academicians, hence this is know to be Industrial Web of Things (IWoT). The main view of Eclipse IoT is to build: The open source developments over IoT standards as well as protocols, Open source schemens and resource that can also be used by IoT solutions and tools designed for IoT developers. [11] [12].

\section{Arduino Yun}

Ardunio Yun as shown in Fig. 3 consists of microcontroller board which works on Linux operating system. It consists of Atmega32u4 processor and Atheros AR9331 processor. The Atmega32u4 processor is supported by Ardunio board and Atheros AR9331 processor which executes over Linux OS. Arduino Yun board contains Wi-Fi, Ethernet, an USB port, microSD card and reset buttons [13] [14].

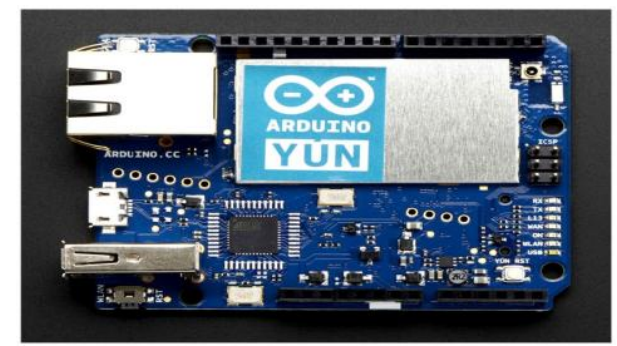

Fig. 3. Arduino Yun [13]

\section{E. BeagleBoard}

BeagleBoard provides a credit card sized board which runs on Android and Linux operating system. It is a low power, single board, open access product developed by TI [Texas Instruments] in association with the Digi-Key and Newark element[14]. In BeagleBoard no simulation softwares are used.

\section{B.Kinoma}

Published By:

Blue Eyes Intelligence Engineering 
BeagleBoard consists of ports such as USB On-The-Go, DVI- D, PC audio, SDHC, JTAG, HDMI and many more. The power consumed by this BeagleBoard is 2 watts. This board works similar to basic computer. BeagleBoard consits of built in storage using PoP chip. [15] [16].

\section{F. Tessel 2}

The Tessel 2 is an open access development board. It works on Java Script and npm. Using Node.JS one can control the working of Tessel 2. This platform can be used for implementing embeded hardwares, testing and tinkering the IoT products. Tessel 2 contains 2 USB ports in which one is conneted to camera and the other to flash storage. This also contains ethernet port, $580 \mathrm{MHz}$ Mediatek router-on- achip, 64MB DDR2 RAM, 802.11 WiFi, $48 \mathrm{MmHz}$ SAMD21coprocessor for making input/output faster as usual. Tessel 2 has a set of Command Line Interface tools which is used to setup and work on Tessel 2 board. [16] [17].

\section{OPEN SOURCE OPERATING SYSTEMS}

In IoT development life cycle, OS can be used to get better results with IoT objects which are inadequate in memory, handling power and quick data transmit over the internet. There are many OS that can be used to get better result. In this section, we have listed few of the OS used in IoT Development lifecycle.

\section{A. AllJoyn}

AllJoyn is one of the operating system for IoT development. It is an openly available operating system intended for IoT formed by means of Linux foundation as well as open connectivity foundation. AllJoyn is flexible and promotes for proximal networks as well as cloud association which is not obligatory. This is originally designed and developed by Qualcomm in association with LG, Panasonic, Microsoft, Sharp, Cisco, Symantec and many more. AllJoyn supports $\mathrm{C}, \mathrm{C}++$, Java, Objective-C, JavaScript backend languages. It is a cross platform API, which can work under various OS like Windows, Linux, Mac, FreeRTOS, Android, iOS operating systems. Some of the services provided by AllJoyn are Onboarding Serivce, Configuration Service, Notification Service, Control Panel Service and CDM [Common Device Model] Service [6] [18] [19].

\section{B. RIOT}

It is a low power embeded operating system. It consists of micro controller architecture and it also allows the application programs such as $\mathrm{C}$ and $\mathrm{C}++$. It runs on 8-bit, 16-bit and 32bit processors. This also provides Portable Operating System Interfare [POSIX]. It also supports standard protocols and network stacks such as UDP, TCP, CoAP, IPv6, 6LoWPAN [20] [21].

\section{Contiki}

Contiki is a free foundation operating system regulation mainly based on Internet of Things. It combines microcon- trollers in simulation with the inter connected objects and it also helps protocols such as RPL, CoAP, IPv6 or 6lowpan as shown in Table I. Some of the features of Contiki operating system are Multitasking kernel, Personal Internet Server, Internet Browser and Graphical End User Interphase [GUI]. Real time applications of Contiki OS are Street Lightning, Radiation Monitoring, Sound Monitoring for Smart Cities, alarms and many more [22] [23].

\section{D.Raspbian}

Raspbian is a card-sized and credit score based operating system developed by Raspberry Pi Foundation works on Unix or Linux operating system. The Kernel type for this operating system is Monolithic. This is no longer used in the current industry beacause of its open source software documentation. It is the well-known family of Raspberry operating system of Linux Environment [24].

TABLE I : Contiki Features [22]

\begin{tabular}{|l|l|}
\hline \multicolumn{1}{|c|}{ Protocols } & \multicolumn{1}{|c|}{ Description } \\
\hline uIP (for IPv4) & $\begin{array}{l}\text { uIP TCP/IP achievement chains 8-bit as well as 16- } \\
\text { bit microcontrollers. }\end{array}$ \\
\hline uIPv6(for IPv6) & uIPv6 is a totally obedient IPv6 extension to uIP. \\
\hline 6LoWPAN & $\begin{array}{l}\text { 6LoWPAN stands for IPv6 in excess of low-power } \\
\text { wireless PAN (personal area networks) It } \\
\text { provides density technology to maintain the } \\
\text { lowrate wireles required by gadgets with }\end{array}$ \\
\hline RPL & $\begin{array}{l}\text { RPL is a distance vector IPv6 standard for LLNs } \\
\text { low-power as well as lossy networks) permits the } \\
\text { simplest dual path to be established in a very ad- } \\
\text { vanced network of gadgets with mixed capability. }\end{array}$ \\
\hline CoAP & $\begin{array}{l}\text { CoAP protocol standard supports communication } \\
\text { for } \\
\text { uncomplicated clear-cut devices, generally }\end{array}$ \\
\hline
\end{tabular}

\section{OPEN SOURCE PROTOCOLS}

The Open Source Protocol initiate implementers on how to access the source code, if the source data is hosted in an open access repository/database on public internet. In this section, we have listed some of the open access protocols used in IoT development life cycle.

\section{A. AMQP}

Advanced Message Queuing Protocol(AMQP) is an open source model and it provides a measured arrangement pattern for industry communication and message-oriented middleware. The significant appearance of AMQP protocol includes routing, message orientation, reliability, ubiquity, interoperability, security, fidelity, manageability, authentication and queuing. AMQP is mainly worn in industrial business communication. One of the advantage of AMQP is, it is a binary wired protocol which helps in message delivering reliably. AMQP communicates mobile information thorough back office information centers. In the IoT era, AMQP is mainly suitable for serverbased investigation functions [25] [26].

\section{B.CoAP}

Constrained Application Protocol(CoAP) might be an internet transmits procedure to be used through stressed nodes and networks. It is considered on behalf of Machine to Machine (M2M) application like well powerd structure automation and it is supported by 
the RequestResponse model between endpoints. Constrained Application Protocol (CoAP) is also a session layer standard designed by IETF controlled RESTful surroundings (CoRE) operational group to afford a light-weight restful interface i,e HTTP. Representational State Transfer (REST) is considered as the common place border between protocol customer as well as server. The main beneficiaries of CoAP are piggy-backed, separate response and non confirmable request as well as response [26] [27].

\section{C.VSCP}

Very Simple Control Protocol(VSCP) is a simple, open source and free protocol for IoT and machine to machine automation. It miles a pretty scalable, extremely low path, unfastened as well as open source for the invention and detection of gadgets. The main configuration of VSCP includes i). Self sustaining tool functionality ii). Updating the overall gadget security iii). A resolution from the sensing element to the end individual requirement [6] [28].

\section{OPEN SOURCE APIS}

Open source APIs plays a major role in IoT development life plan. Open access APIs provides a platform for information collection, processing, visualization as well as device management. In this section, we have listed some of the APIs used in IoT development life cycle.

\section{A. Zetta}

Zetta is a free release supply board made of Node.js for developing Web over Things. Using Zetta private servers will lead throughout Geo-distributed worldwide computers or the private cloud. Zetta consists of REST APIs, WebSockets or effective planning because of its aggregation towards gadgets between data-intensive and real-time applications [29] [30].

\section{B.BipIO}

BipIO is an easy-to-access Hyper Text Transfer Protocol API. This can be used for drag-and-drop visualization to join Web APIs together with many objects. BipIO is surprisingly balance node.js based API integration. It makes use of graph based pipelines or 'Bips' in conformity with endpoints. Bips comes in a variety of flavors such as WebHooks/Sockets, Email or work Triggers. It is a RESTful JSON API that wires description stage named spacing and several domains for every account. Consumers authenticate under HTTP Basic. BipIO can be installed next to your presented open source application or model for out-of-band communication conversion. This provides an aggregation, queuing, social association. Some of the features of BipIO includes:

- Self-motivated or robotically derived naming.

- Pause or self-destructing behind a confident time or imitation volume.

- Links customers with soft ACLs under the path of their 'life'.

- Able to be reconfigured dynamically without changing a client implementation .
- Substantially extensible, as of any channel to any additional channel. [31]

\section{OPEN SOURCE NODE FLOW EDITORS}

Node flow editor are the fundamental easy to use programming tools for wiring hardware devices, APIs and cloud services in new way. In this section, we have listed popular node flow editors for IoT development life cycle.

\section{A. Node-RED}

Node-RED being a stream oriented Internet of Things encoding device for relating hardware gadgets, APIs as well as online web services in an attractive and innovative way. Nodes preserve IoT gadgets, network APIs, cloud services or every building block with the purpose of approving and sending messages. Nodes encapsulate composite building blocks such as databases, APIs, protocol handlers, logic, and many more. The properties of nodes are able to easily extend by installing novel nodes produced by the public community and the flows that one can build and share as JSON document as shown in the Fig. 4. While, the majority programming in Node-RED is programmed visually by predefined methods and extra features can be added in JavaScript. The main features of Node-Red are as listed in Table II [32] [33].

TABLE II: Node-Red Features [33]

\begin{tabular}{|l|l|}
\hline \multicolumn{1}{|c|}{ Types } & \multicolumn{1}{c|}{ Description } \\
\hline Node-RED & $\begin{array}{l}\text { A visual tool for wiring the Internet of } \\
\text { Things }\end{array}$ \\
\hline Node-RED & A dashboard UI for Node-RED \\
\hline Node generator & $\begin{array}{l}\text { Command line tool to generate } \\
\text { Node-RED node modules from } \\
\text { several various sources, including } \\
\text { Open API document and function } \\
\text { node's source. }\end{array}$ \\
\hline $\begin{array}{l}\text { Node-RED } \\
\text { CommandLine } \\
\text { Tool }\end{array}$ & $\begin{array}{l}\text { Command-line tool allows you to } \\
\text { remotely administer a Node-RED } \\
\text { instance. }\end{array}$ \\
\hline $\begin{array}{l}\text { Node-RED } \\
\text { Pre-installed } \\
\text { devices }\end{array}$ & $\begin{array}{l}\text { Raspberry Pi,IoT Gateway,Predix } \\
\text { Developer Kit. }\end{array}$ \\
\hline $\begin{array}{l}\text { Node-RED } \\
\text { Cloud } \\
\text { services }\end{array}$ & $\begin{array}{l}\text { Cisco cloud,IBM Cloud,AT and T IoT } \\
\text { Platform. }\end{array}$ \\
\hline
\end{tabular}



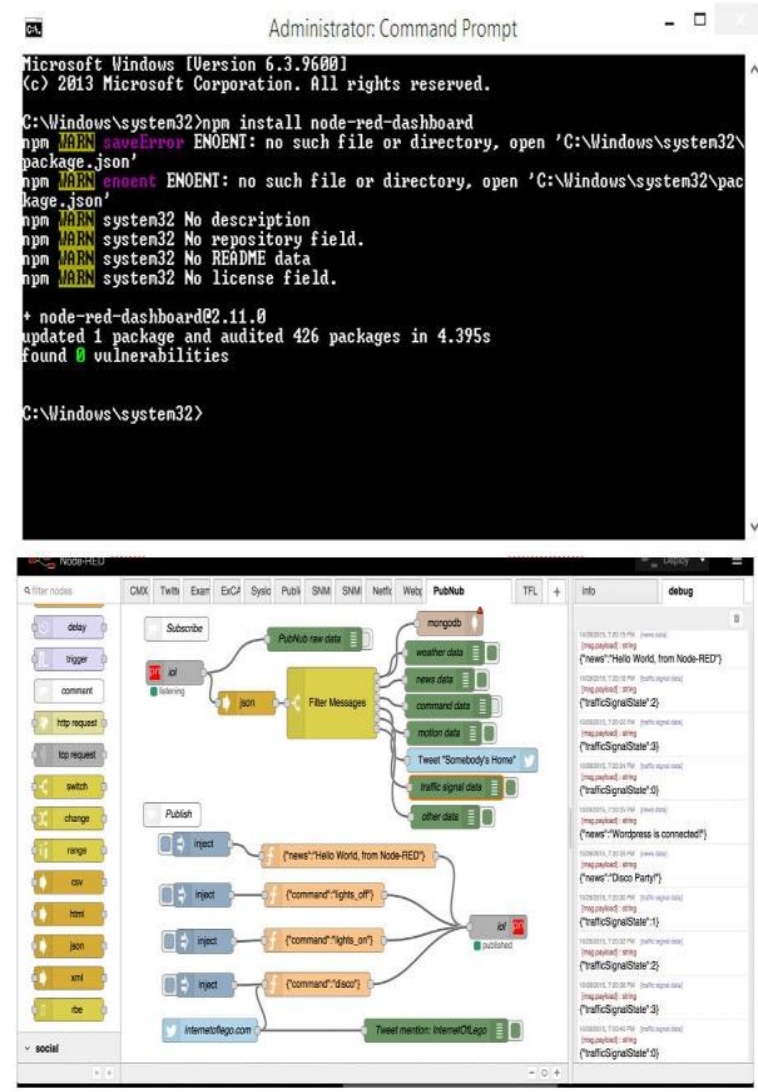

Fig. 4. Node-Red Command Promt and Flow Editor [32]

\section{B.ThingBox}

ThingBox contains a set of readymade softwares and it should be installed as well as configured on SDCard. The ThingBox is a ready to use software. The main drawback of this utensil is far behind the home automation. The most important endeavor of this tool is to implement the novel use case with the intention of home automation as shown in Fig. 5 [6] [34].

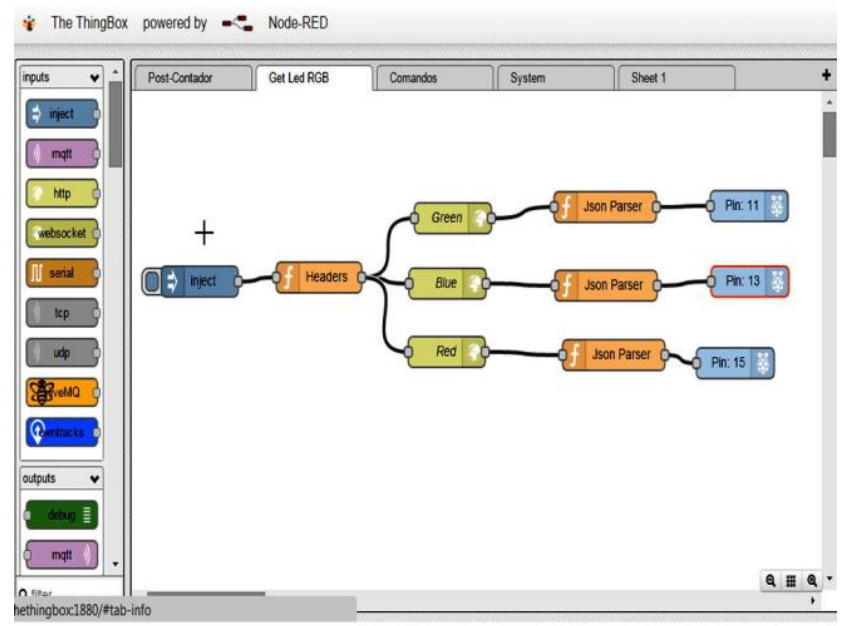

Fig. 5. Node-Red MQTT Using ThingBox [34]

\section{OPEN SOURCE DATA VISUALIZATION}

open source data visualization tools help to process, cleanse and display the rapidly growing complex information collected every second. This supports the decision making and data analytic techniques for collected information.

\section{A. ThingSpeak}

ThingSpeak is an Internet of Things analytical tool which examines the intention of aggregate, outlook and determines the existance of information data stream from the cloud. It gives instantaneous visualization of statistics information sent to ThingSpeak from device objects. ThingSpeak is a functional proposal in favor of Internet of Things. The ThingSpeak will allow building a function more or less statistics composed by sensors. The main characteristic of ThingSpeak are real-time information gathering, information processing, visualizations, applications, and plug-ins. ThingSpeak collects the information from Hardware devices like Ardino and Raspberry pi [35] [36].

\section{B. Freeboard}

FreeBoard as shown in Fig. 6 is a simple and powerful dashboard build for real-time, interaction and visualizations using drag and drop interface. It is affordable to very less price with open source. The main features of FreeBoard are:

- Flexible data source

- Drag and drop interface

- Develop with widgets

- Convert individual look and feel

- Public or private access

- Clone it

- Share instantly to the cloud [37] [38].

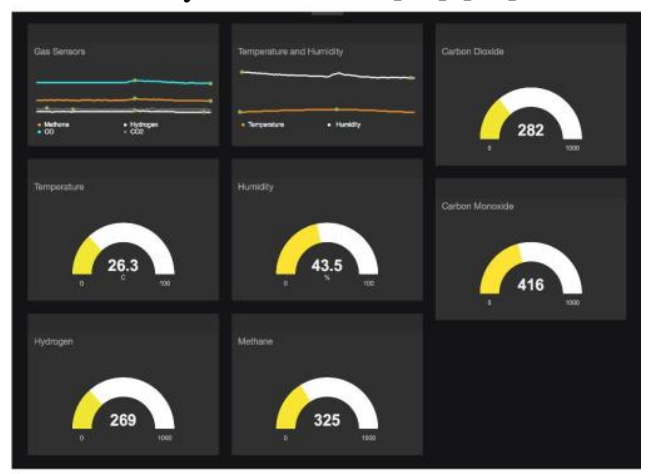

Fig. 6. Output Graph Using FreeBoard Tool [38]

\section{OPEN SOURCE MIDDLEWARE}

Open source middleware softwares sits between the OS and application layer. This provides communication services for different applications that can connect with each other.

\section{A.OpenIoT}

It is a world wide web explains the development of open free foundation middleware which supports sensor cloud data. It aims to facilitate cloud-based Sensing-as-a-Service to implement intellectual agriculture, elegant manufacture, urban bunch sensing, elegant living as well as smart campuses. This middleware can maintain versatile configuration and exploitation of algorithms for assortment and filtering data streams by stemming on or after the web-connected substance [39] [40]. 


\section{B.IoTSyS}

IoTSyS is a middleware integration for web of things. It sup- ports a communication stack such as IPv6, oBIX, 6LoWPAN, Constrained Application Protocol and active XML Interchange standards as well as protocols standards. Open-IoT is not only integration middleware because of existence of IoTSyS in web of Things. The IoTSyS provides a verbal exchange stack implanted procedure based IPv6, Internet services, oBIX according to grant interoperable interface because of clever objects. The predominant objective about IoTSyS is in accor- dance that leverage the limit over current automation systems or sensors. It effortlessly sets up an instant solution, addressing security, discovery and scalability troubles [41].

\section{RESULTS AND DISCUSSIONS}

Open root segregation tools and its associated platforms is an end to end application security platform in IoT development lifecycle.

\section{A. DeviceHive}

DeviceHive offer a contact configuration intended for connect- ing M2M tactic in the direction of the Internet of Things. It includes user-friendly internet association software system for network, submission security policy as well as live observance approach. It additionally contains IDs on sample projects implemented with DeviceHive and contains simulation phase wherever and whenever DeviceHive provides visualization works on-line [42] [43].

\section{B. DeviceHub.net}

DeviceHub.net will be measured based on open-root spinal column of Web of Things. It facilitates a private cloud ca pability to observe, track, deal with the devices and provides information composed from internet in a straight line and in real time period. It is primarily involved concerning about wellness precaution, positioning kids on transport and atmo spheric condition [44].

\section{CONCLUSION}

The study analyzes the open source tools for IoT applica-tions. Open source is a set up actuality among the Internet of Things. It is already chronic by means of the substantial majority of IoT developer and nine out of ten uses open source application. At least 6 among 10 throw in to open source tasks as well. In similar, all can perform high-quality open source option designed for any sort of device and technology used by implementer. Open supply is a path in conventionality with gain efficiencies as well as stimulate innovation via non- differentiate components and technological stack [45].

\section{REFERENCES}

[1] P. S. Misra, "Introduction to internet of things," ttps://nptel.ac.in/ content/storage2/nptel data3/html/mhrd/ict/text/106105166/lec2.pdf, [online].

[2] V. Gazis, M. Goertz, M. Huber, A. Leonardi, K. Mathioudakis, A. Wiesmaier, and F. Zeiger, "Short paper: Iot: Challenges, projects, architectures," in 2015 18th International Conference on Intelligence in Next Generation Networks. IEEE, 2015, pp. 145-147.
[3] P. P. Ray, "A survey of iot cloud platforms," Future Computing and Informatics Journal, vol. 1, no. 1-2, pp. 35-46, 2016.

[4] D. S. Balakrishnan, "A quick look at open source tools and services foriot,"https://opensourceforu.com/2019/03/a-quick-look-at-open-sour ce- tools-and-services-for-iot/, [online].

[5]https://robu.in/product/arduino-uno-r3-ch340g-atmega328pdevlopmentboard/, [online].

[6]https://www.sam-solutions.com/blog/the-best-tools-for-internetofthings-iot-development/, [online].

[7] https://en.wikipedia.org/wiki/Arduino, [online].

[8] S. Athani, C. Tejeshwar, M. M. Patil, P. Patil, and R. Kulkarni, "Soil moisture monitoring using iot enabled arduino sensors with neural networks for improving soil management for farmers and predict seasonal rainfall for planning future harvest in north karnatakaindia," in 2017 International Conference on I-SMAC (IoT in Social, Mobile, Analytics and Cloud)(I-SMAC). IEEE, 2017, pp. 43-48.

[9] https://www.prnewswire.com/news-releases/marvell-unvei ls-kinomacreate-the-javascript-powered-maker-kit-for-bu ilding-internet-ofthings-projects\%96prototyping-consum er-electronics-249261081.html

[10] https://en.wikipedia.org/wiki/Kinoma, [online].

[11] https://wiki.eclipse.org/IoT., [online].

[12] M. Aly, F. Khomh, and S. Yacout, "Kubernetes or openshift? which technology best suits eclipse hono iot deployments," in 2018 IEEE 11th Conference on Service-Oriented Computing and Applications (SOCA). IEEE, 2018, pp. 113-120.

[13] "Getting started with the arduino yn," https://www.arduino.cc/en/Guide/ ArduinoYun, [online].

[14] D. Bruneo, S. Distefano, F. Longo, G. Merlino, A. Puliafito, and A. Zaia, "Head in a cloud: An approach for arduino yun virtualization," in 2017 Global Internet of Things Summit (GIoTS). IEEE, 2017, pp. 1-6.

[15] https://en.wikipedia.org/wiki/BeagleBoard, [online].

[16] A. Raza, A. A. Ikram, A. Amin, and A. J. Ikram, "A review of low cost and power efficient development boards for iot applications," in 2016 Future Technologies Conference (FTC). IEEE, 2016, pp. 786-790.

[17] https://tessel.io/, [online].

[18] https://en.wikipedia.org/wiki/AllJoyn, [online]

[19] O. Tomanek and L. Kencl, "Security and privacy of using alljoyn iot framework at home and beyond," in 2016 2nd international conference on intelligent green building and smart grid (IGBSG). IEEE, 2016, pp. $1-6$.

[20] https://en.wikipedia.org/wiki/RIOT-operating-system, [online].

[21] E. Baccelli, C. Gündoğan, O. Hahm, P. Kietzmann, M. S. Lenders,

H. Petersen, K. Schleiser, T. C. Schmidt, and M. Wählisch, "Riot: An open source operating system for low-end embedded devices in the iot," IEEE Internet of Things Journal, vol. 5, no. 6, pp. 4428-4440, 2018.

[22] https://en.wikipedia.org/wiki/Contiki, [online].

[23] M. Malik, M. Dutta et al., "Contiki-based mitigation of udp flooding attacks in the internet of things," in 2017 International Conference on Computing, Communication and Automation (ICCCA). IEEE, 2017, pp. 1296-1300.

[24] G. O. E. Abdalla and T. Veeramanikandasamy, "Implementation of spy robot for a surveillance system using internet protocol of raspberry pi," in 2017 2nd IEEE International Conference on Recent Trends in Electronics, Information \& Communication Technology (RTEICT) IEEE, 2017, pp. 86-89.

[25]“Advancedmessagequeuingprotocol,'https://www.iotone.com/term/ advanced-message-queuing-protocol-amqp/t35, [online].

[26] N. Naik, "Choice of effective messaging protocols for iot systems: Mqtt, coap, amqp and http," in 2017 IEEE international systems engineering symposium (ISSE). IEEE, 2017, pp. 1-7.

[27] F. Azzola, "Coap protocol: Step-by-step guide," articles / coap -protocol-step-by-step-guide, [online].

[28] https://www.vscp.org/, [online].

[29] https://www.w3.org/WoT/IG/wiki/images/9/95/The essential guide to Open Source in IoT - White Paper.pdf, [online]

[30] https://www.zettajs.org/, [online].

[31] https://iotbyhvm.ooo/iot-open-source-development-tools/, [online].

[32] M. Lekić and G. Gardašević, "Iot sensor integration to node-red platform," in 2018 17th International Symposium INFOTEH-JAHORINA (INFOTEH). IEEE, 2018, pp. 1-5.

[33] https://en.wikipedia.org/wiki/Node-RED, [online]. [34] http://thethingbox.io/, [online]. 
[35] K. Sabanc1, E. Yigit, D. Üstün, A. Toktaş, and Y. Çelik, "Thingspeak based monitoring iot system for counting people in a library," in 2018 International Conference on Artificial Intelligence and Data Processing (IDAP). IEEE, 2018, pp. 1-6.

[36]https://community.thingspeak.com/tutorials/arduino/send-data -to- thingspeak-with-arduino/, [online].

[37] R. R. Rajanna, S. Natarajan, and P. R. Vittal, "An iot wi-fi connected sensor for real time heart rate variability monitoring," in $20183 \mathrm{rd}$ International Conference on Circuits, Control, Communication and Computing (I4C). IEEE, 2018, pp. 1-4.

[38] https://freeboard.io/, [online].

[39] J. Kim and J.-W. Lee, "Openiot: An open service framework for the internet of things," in 2014 IEEE world forum on internet of things (WF-IoT). IEEE, 2014, pp. 89-93.

[40] http://brookscanavesi.com/blog/top-10-open-source-tools-iot -application-development/

[41] https://devicehive.com/, [online].

[42] M. Lyaskov, G. Spasov, and G. Petrova, "A practical implementation of smart home energy data storage and control application based on cloud services," in 2017 XXVI International Scientific Conference Electronics (ET). IEEE, 2017, pp. 1-4.

[43] http://opensourcetour.com/2018/10/the-top-open-source-iot-platform s-for-development[online]

[44]https://devicehub.net [online].

[45] https://www.w3.org/WoT/IG/wiki/images/9/95/The ess ential_guide_to_Open_Source_in_IoT___White_Paper. pdf

\section{AUTHORS PROFILE}

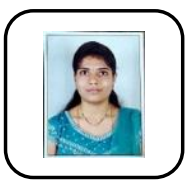

Shilpa V, is currently working as Assistant Professor in Department of Computer Science and Engineering at $\mathrm{C}$ Byregowda Institute of Technology, Kolar,India. She received her Bachelor of Engineering degree in Information Science and Engineering from Visvesvaraya Technological University, Belagavi and Master of Engineering degree in Computer Networking from University Visvesvaraya College of Engineering, Bangalore University, Bengaluru. She is currently pursuing Ph.D under the guidance of Dr.Vidya A in Computer Science and Engineering at Vivekananda Institute of Technology, affiliated to Visvesvaraya Technological University, Bangalore, India . Her research interest includes Internet of Things, with a focus on security and authentication protocols, Cloud Computing.

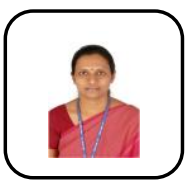

Dr. Vidya A, is currently working as Professor and Head of the Department of Computer Science and Engineering at Vivekananda Institute of Technology Bangalore, India. She received her Bachelor of Engineering and Masters of Engineering degrees in Computer Science and ngineering from Bangalore University, Bangalore. She was awarded Ph.D. in Computer Science and Engineering from Jawaharlal Nehru Technological University, Hyderabad. Her area of research includes Data Mining, Soft Computing, Pattern Recognition, Computer Networks and Image Processing. She is a Life Member of Indian Society for Technical Education, Computer Society of India and International Association of Engineers. She is a Fellow of Institute of Engineers.

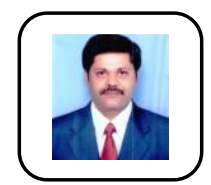

Dr. S N Chandrashekara, is currently working as Professor and Head of the Department of Computer Science and Engineering at C Byregowda Institute of Technology, Kolar, India. He received his Bachelor of Engineering from Bangalore University and Masters of Technology in Computer Science and Engineering from NITK, Suratkal. He was awarded Ph.D. in Network and Devices from IISc, Bangalore. His area of research includes Computer Networks,Cloud Computing, Cyber Security and IOT Medical Image Processing. He is a Life Member of Indian Society for Technical Education, Computer Society of India. He is a Fellow of Institute of Engineers and Life member of ISSE. 